\title{
Limit-point Classification for Singular Conformable Fractional Sturm-Liouville Operators
}

\author{
Bilender P. Allahverdiev ${ }^{1}$ (D), Hüseyin Tuna ${ }^{2}$ (D), Yüksel Yalçinkaya ${ }^{1, *}$ (D) \\ ${ }^{1}$ Department of Mathematics, Faculty of Art and Sciences, Süleyman Demirel University, 32260, Isparta, Turkey. \\ ${ }^{2}$ Department of Mathematics, Faculty of Art and Sciences, Mehmet Akif Ersoy University, 15030, Burdur, Turkey.
}

Received: 09-11-2020 • Accepted: 16-01-2021

Aвstract. In this work, we study the following conformable fractional Sturm-Liouville problem

$$
l[y]=-T_{\alpha}\left(p(t) T_{\alpha} y(t)\right)+q(t) y(t),
$$

where $t \in[0, \infty)$, the real-valued functions $p$ and $q$ satisfy the following conditions:

$$
\begin{gathered}
\text { (i) } \quad q \in L_{\alpha}^{2}[0, \infty), \\
\text { (ii) } p \text { is absolutely continuous on }[0, \infty), \\
\text { (iii) } \quad p(t)>0 \text { for all } t \in[0, \infty)
\end{gathered}
$$

The conformable fractional Sturm-Liouville problem is of the limit-point case if the number of linearly independent $\alpha$-square integrable solutions of the equation $l[y]=\lambda y$ is less than 2 . We give a criterion for the limit point classification of conformable fractional Sturm-Liouville operators in singular case.

2010 AMS Classification: 34L05, 47E05

Keywords: Sturm-Liouville operator, conformable fractional Sturm-Liouville operator, limit-point case.

\section{INTRODUCTION}

Differential equations; it is used as a mathematical model in many different sciences such as physics, chemistry, engineering, biology, and economics. Newton and Leibnitz studied differential and integral calculus in the second half of the 17th century. Due to the limited application of fractional derivatives and problem-solving, it was not possible to make sufficient studies on this subject in the 17 th century. With the discovery of the fractional calculus technique; many problems such as chaos, propagation and wave motions, filtering and irreversibility can be modeled and explained more accurately using fractional analysis. This interesting topic is used in fields such as diffusion, Schrödinger equation, materials science, transmission lines theory, chemical analysis of liquids, heat transfer, fluids, biology, biophysics, bioengineering, electromagnetic theory, mechanics, physics and control theory, analytical and numerical methods. The day comes out with new and interesting applications. Besides, the use of fractional calculation to model the physical states of viscoelastic (adhesive and flexible) materials (cartilage, skin, muscle) in which hysteresis, memory and tension factors occur naturally occurs spontaneously [10-13]. 
In recent years, conformable fractional derivative and conformable fractional integral were defined by Khalil and his friends. In their study, they presented a linearity requirement for the new definition of fractional derivative, multiplication rule, division rule, fractional Rolle theorem and fractional average value theorem for conformable fractional derivative [7]. Later in [1], Abdeljawad defined the right and left conformable fractional derivatives, the fractional chain rule and fractional integrals of higher orders.

Conformable fractional derivative aims to expand the derivative definition as known by providing the natural characteristics of classical derivative and to pain new perspectives for differential equation theory with the help of conformable differential equations obtained as using this derivative definition [8].

The Levinson criterion is considered to be one of the most important limit-point criteria for the differential operator, since it provides a more general boundary point criterion [9]. Baleanu et al. [3] gave the Levinson criterion for 2 $\alpha$-order conformable fractional Sturm-Liouville operator. Zhaowen et al. studied the $2 \alpha$-order Sturm-Liouville operator with conformable fractional derivatives. They have obtained two limit-point case criteria for this operator [15].

In this study, we shall give a criterion for the limit-point classification of conformable fractional Sturm-Liouville operators in singular case. While proving our results, we use the machinery and methods of [4-6].

Now, some preliminary concepts and theorems are presented for the convenience of the reader.

Definition 1.1 ( [1]). Let $0<\alpha<1$. For a function $f:(0, \infty) \rightarrow \mathbb{R}:=(-\infty, \infty)$, the conformable derivative of order $\alpha$ of $f$ at $t>0$ is defined by

$$
T_{\alpha} f(t)=\lim _{\varepsilon \rightarrow 0} \frac{f\left(t+\varepsilon t^{1-\alpha}\right)-f(t)}{\varepsilon},
$$

and the conformable derivative at 0 is defined by

$$
\left(T_{\alpha} f\right)(0)=\lim _{t \rightarrow 0^{+}}\left(T_{\alpha} f(t)\right) .
$$

Definition 1.2 ( [1]). The left conformable derivative of order $\alpha$ of a function $f:[a, \infty) \rightarrow \mathbb{R}$ is defined by

$$
\left(T_{\alpha}^{a} f\right)(t)=\lim _{\varepsilon \rightarrow 0} \frac{f\left(t+\varepsilon(t-a)^{1-\alpha}\right)-f(t)}{\varepsilon}, 0<\alpha \leq 1 .
$$

When $a=0$ we write $T_{\alpha}$. If $\left(T_{\alpha} f\right)(t)$ exists on $(a, b)$ then

$$
\left(T_{\alpha}^{a} f\right)(a)=\lim _{t \rightarrow a^{+}}\left(T_{\alpha}^{a} f\right)(t) .
$$

Definition 1.3 ( [1]). The right conformable derivative of order $\alpha$ of $f:(-\infty, b]$ is defined by

$$
\left({ }_{\alpha}^{b} T f\right)(t)=-\lim _{\varepsilon \rightarrow 0} \frac{f\left(t+\varepsilon(b-t)^{1-\alpha}\right)-f(t)}{\varepsilon},
$$

where $0<\alpha \leq 1$ and $\left({ }^{b} T_{\alpha} f\right)(b)=\lim _{t \rightarrow b^{-}}\left({ }^{b} T_{\alpha} f\right)(t)$.

Theorem 1.4 ( [1]). Let $x, y:[0, b] \rightarrow \mathbb{R}$ be two functions such that $x$ and $y$ are conformable fractional differentiable. Then, we have

$$
\int_{0}^{b} y(t) T_{\alpha}(x)(t) d_{\alpha} t+\int_{0}^{b} x(t) T_{\alpha}(y)(t) d_{\alpha} t=x(b) y(b)-x(0) y(0) .
$$

Definition 1.5 ( [2]). Let $L_{\alpha}^{2}[0, \infty)$ be the space of all complex-valued functions defined on $[0, \infty)$ such that

$$
\|y\|:=\sqrt{\int_{0}^{\infty}|y(t)|^{2} d_{\alpha} t}=\sqrt{\int_{0}^{\infty}|y(t)|^{2} t^{\alpha-1} d_{\alpha} t}<\infty .
$$

The space $L_{\alpha}^{2}[0, \infty)$ is a Hilbert space with the inner product

$$
\langle y, z\rangle:=\int_{0}^{\infty} y(t) \overline{z(t)} d_{\alpha} t, \text { where } y, z \in L_{\alpha}^{2}[0, \infty)
$$

Definition 1.6 ( [6]). Two functions $f, g$ will be said to be effectively proportional if there are constants $\beta_{1}, \beta_{2}$, not both zero, such that $\beta_{1} f \equiv \beta_{2} g$. A null function is effectively proportional to any function. 
Theorem 1.7. $i$ ) If $\beta_{1}, \beta_{2}, \ldots, \beta_{n}$ are positive and $\beta_{1}+\beta_{2}+\ldots+\beta_{n}=1$, then

$$
\int\left|f^{\beta_{1}} g^{\beta_{2}} \ldots l^{\beta_{n}}\right| d_{\alpha} t<\left(\int|f| d_{\alpha} t\right)^{\beta_{1}}\left(\int|g| d_{\alpha} t\right)^{\beta_{2}} \ldots\left(\int|l| d_{\alpha} t\right)^{\beta_{n}},
$$

unless one of the functions is null or all are effectively proportional.

ii) If $k>1$ and $\frac{1}{k}+\frac{1}{k^{\prime}}=1$, then

$$
\int|f g| d_{\alpha} t<\left(\int|f|^{k} d_{\alpha} t\right)^{\frac{1}{k}}\left(\int|g|^{k^{\prime}} d_{\alpha} t\right)^{\frac{1}{k^{\prime}}}
$$

unless either (a) $f^{k}$ and $g^{k^{\prime}}$ are effectively proportional or (b) $\mathrm{fg}$ is null.

Proof. See [6], as the proof is unchanged except for minor notational modifications.

Theorem 1.8. If $p>1, f \in L_{\alpha}^{p}(0, a)$, and

$$
F(x)=\int_{0}^{x}|f(t)| d_{\alpha} t
$$

then we have

$$
F(x)=o\left(\left[\frac{x}{\alpha}\right]^{\frac{\alpha}{p^{\prime}}}\right)
$$

for small $x$.

Proof. It follows from Theorem 1.7 that

$$
F^{p} \leqq \int_{0}^{x}|f|^{p} d_{\alpha} t\left(\int_{0}^{x} d_{\alpha} t\right)^{p-1}=\frac{x^{\alpha(p-1)}}{\alpha} \int_{0}^{x}|f|^{p} d_{\alpha} t
$$

and the second factor tends to 0 .

Theorem 1.9. If $p>1$ and $f \in L_{\alpha}^{p}(0, \infty)$, then $F(x)=o\left(\left[\frac{x}{\alpha}\right]^{\frac{\alpha}{p^{\prime}}}\right)$ both for small and for large $x$.

Proof. For small $x$, the proof is obtained by Theorem 1.8. Now, we prove the result for large $x$. Choose $\eta$ so that

$$
\int_{\eta}^{\infty}|f|^{p} d_{\alpha} x<\varepsilon^{p}
$$

and suppose $x>\eta$. Then, we obtain

$$
\begin{aligned}
&(F(x)-F(\eta))^{p}=\left(\int_{\eta}^{x}|f| d_{\alpha} t\right)^{p} \leqq \frac{(x-\eta)^{\alpha(p-1)}}{\alpha} \int_{\eta}^{x}|f|^{p} d_{\alpha} t<\frac{\varepsilon^{p} x^{(p-1) \alpha}}{\alpha}, \\
& F(x)<F(\eta)+\varepsilon \frac{x^{\frac{\alpha}{p^{\prime}}}}{\alpha}<2 \varepsilon \frac{x^{\frac{\alpha}{p^{\prime}}}}{\alpha}
\end{aligned}
$$

for sufficiently large $x$.

\section{Main Results}

We consider the following conformable fractional singular Sturm-Liouville expression

$$
l(y)=-T_{\alpha}\left(p(t) T_{\alpha} y(t)\right)+q(t) y(t) \text { on }[0, \infty),
$$

where the real-valued functions $p$ and $q$ satisfy the following conditions:

(i) $q \in L_{\alpha}^{2}[0, b]$ for all $b>0$,

(ii) $p$ is absolutely continuous on $[0, b]$ for all $b>0$,

(iii) $\quad p(t)>0$ for all $t \in[0, \infty)$. 
The set $D$ is defined by: $y \in D$ if

$$
\begin{aligned}
& \text { (i) } y \in L_{\alpha}^{2}[0, \infty), \\
& \text { (ii) } T_{\alpha} y \text { is absolutely continuous on }[0, b] \text { for all } b>0 \text {, } \\
& \text { (iii) } l(y) \in L_{\alpha}^{2}[0, \infty), \\
& \text { (iv) } y(0)=0 .
\end{aligned}
$$

For $y_{1}, y_{2} \in D$, we have the following Green's formula [2]

$$
\int_{0}^{\infty} l\left(y_{1}\right)(t) \overline{y_{2}(t)} d_{\alpha} t-\int_{0}^{\infty} y_{1}(t) \overline{l\left(y_{2}\right)(t)} d_{\alpha} t=\left[y_{1}, y_{2}\right](\infty)-\left[y_{1}, y_{2}\right](0),
$$

where

and

$$
\left[y_{1}, y_{2}\right](t)=p(t)\left\{y_{1}(t) \overline{T_{\alpha} y_{2}(t)}-T_{\alpha} y_{1}(t) \overline{y_{2}(t)}\right\}, t \in[0, \infty)
$$

$$
\left[y_{1}, y_{2}\right](\infty):=\lim _{t \rightarrow \infty}\left[y_{1}, y_{2}\right](t) .
$$

Theorem 2.1. If the function $q$ is bounded below on $[0, \infty)$ and

$$
\int_{0}^{\infty}\{p(t)\}^{-\frac{1}{2}} d_{\alpha} t<\infty
$$

then the differential operator l defined by (2.1) is in the limit-point case at infinity.

Proof. It is known, see ( $[4,14])$, that $l$ is limit-point at infinity if and only if

$$
\left[y_{1}, y_{2}\right](\infty)=0 \text {. }
$$

for all $\in D$. Hence it is sufficient to prove that, for all $y_{1}, y_{2} \in D$ (where $y_{1}$ and $y_{2}$ are real-valued functions),

$$
\lim _{b \rightarrow \infty} p(b) y_{1}(b) T_{\alpha} y_{2}(b)=0 .
$$

Without loss of generality we may assume that there is a positive constant $k$ such that

$$
q(t) \geq k>0 \text { for all } t \in[0, \infty)
$$

From (2.6), for all $b>0$, we get,

(2.7) implies that

$$
Q(b)=\int_{0}^{b} q(t) d_{\alpha} t \geq k \frac{b^{\alpha}}{\alpha}
$$

$$
[Q(b)]^{-1 / 2} \leq\left[k \frac{b^{\alpha}}{\alpha}\right]^{-1 / 2}
$$

for all $b>0$.

By integration by parts (1.1), we obtain

$$
\int_{0}^{b}\left\{p T_{\alpha} y_{1} T_{\alpha} y_{2}+q y_{1} y_{2}\right\} d_{\alpha} t=\left.\left[p y_{1} T_{\alpha} y_{2}\right]\right|_{0} ^{b}+\int_{0}^{b} l\left(y_{2}\right) y_{1} d_{\alpha} t,
$$

for all $y_{1}, y_{2} \in D$ and for all $b>0$.

If we take $y_{1}=y_{2} \in D$ in (2.9), then we get

$$
\int_{0}^{b}\left\{p\left(T_{\alpha} y_{1}\right)^{2}+q y_{1}^{2}\right\} d_{\alpha} t=p(b) y_{1}(b) T_{\alpha} y_{1}(b)-\int_{0}^{b} l\left(y_{1}\right) y_{1} d_{\alpha} t .
$$

It follows from (2.2) and (2.6) that the integrand on the left is non-negative. From (2.3), the integrand on the right is $L_{\alpha}^{2}[0, \infty)$. If $p\left(T_{\alpha} y_{1}\right)^{2}+q y_{1}^{2} \notin L_{\alpha}^{1}[0, \infty)$, then $p(b) y_{1}(b) T_{\alpha} y_{1}(b) \rightarrow \infty$, as $b \rightarrow \infty$. This is impossible since then both $y_{1}(b)$ and $T_{\alpha} y_{1}(b)$ would have the same sign for all large $b$ and $y_{1}$ could not then belong to $L_{\alpha}^{2}[0, \infty)$. This contradicts our assumption $y_{1} \in D \subset L_{\alpha}^{2}[0, \infty)$. Thus, for all $y_{1} \in D$, we conclude that

$$
p^{\frac{1}{2}} T_{\alpha} y_{1} \in L_{\alpha}^{2}[0, \infty), q^{\frac{1}{2}} y_{1} \in L_{\alpha}^{2}[0, \infty) .
$$


From (2.9), we deduce that, for all $y_{1}, y_{2} \in D$,

$$
\lim _{b \longrightarrow \infty} p(b) y_{1}(b) T_{\alpha} y_{2}(b)
$$

exists and is finite.

If $\Psi \in L_{\alpha}^{2}[0, \infty)$, then $\Psi \in L_{\alpha}^{1}[0, b]$. It follows from Theorem 1.9 that

$$
\lim _{b \rightarrow \infty}\left(\frac{b}{\alpha}\right)^{-\frac{1}{2} \alpha} \int_{0}^{b}|\Psi(t)| d_{\alpha} t=0
$$

For all $b \geq 0$, define

By (2.4), we get

$$
P(b):=\int_{0}^{b}\{p(t)\}^{-\frac{1}{2}} d_{\alpha} t .
$$

$$
\lim _{b \longrightarrow \infty} P(b)=K \text {, where } 0<K<\infty .
$$

From (1.1), we conclude that

$$
\begin{gathered}
\int_{0}^{b} p(t)^{\frac{1}{2}} T_{\alpha} y_{2}(t) d_{\alpha} t=P(b) p(b) T_{\alpha} y_{2}(b) \\
+\int_{0}^{b} P(t) l\left(y_{2}(t)\right) d_{\alpha} t-\int_{0}^{b} P(t) q(t) y_{2}(t) d_{\alpha} t .
\end{gathered}
$$

Multiply this result by $\{Q(b)\}^{-\frac{1}{2}}$ and consider the separate terms. it follows from (2.8), (2.10) and (2.12) that

$$
\begin{gathered}
\{Q(b)\}^{-\frac{1}{2}} \int_{0}^{b} p(t)^{\frac{1}{2}} T_{\alpha} y_{2}(t) d_{\alpha} t= \\
O\left(\left(\frac{b}{\alpha}\right)^{-\frac{\alpha}{2}} \int_{0}^{b} p(t)^{\frac{1}{2}}\left|T_{\alpha} y_{2}(t)\right| d_{\alpha} t\right)=o(1) \text { as } b \rightarrow \infty .
\end{gathered}
$$

Similarly, using (2.8), (2.10) and (2.12) as $b \rightarrow \infty$, we get

$$
\begin{aligned}
& \{Q(b)\}^{-\frac{1}{2}} \int_{0}^{b} P(t) l\left(y_{2}(t)\right) d_{\alpha} t \\
& =O\left(K\left(\frac{b}{\alpha}\right)^{-\frac{\alpha}{2}} \int_{0}^{b}\left|l\left(y_{2}\right)\right| d_{\alpha} t\right)=o(1) .
\end{aligned}
$$

Let $b^{\prime}>0$ be fixed. Then, for all $b>b^{\prime}$, we have

$$
\begin{gathered}
Q(b)^{-\frac{1}{2}} \int_{0}^{b} P(t) q(t) y_{2}(t) d_{\alpha} t \\
=Q(b)^{-\frac{1}{2}}\left\{\int_{0}^{b^{\prime}}+\int_{b^{\prime}}^{b}\right\}\left(P q y_{2}\right) d_{\alpha} t=O\left(b^{-\frac{1}{2}}\right) \\
+O\left(K\left\{Q(b)^{-1} \int_{b^{\prime}}^{b} q d_{\alpha} t \int_{b^{\prime}}^{b} q y_{2}^{2} d_{\alpha} t\right\}^{\frac{1}{2}}\right) \\
=o(1)+O\left(K\left\{\int_{b^{\prime}}^{b} q y_{2}^{2} d_{\alpha} t\right\}^{\frac{1}{2}}\right)
\end{gathered}
$$

It follows from (2.10) that the left-hand side tends to zero as $b \rightarrow \infty$. 
Hence by (2.13), we get, for all $y_{2} \in D$,

$$
\lim _{b \rightarrow \infty}\{Q(b)\}^{-\frac{1}{2}} p(b) T_{\alpha} y_{2}(b)=0 .
$$

Let us consider $\{Q\}^{\frac{1}{2}} y_{1}$ where $y_{1} \in D$; assume that

$$
\lim _{b \rightarrow \infty} \inf \{Q(b)\}^{\frac{1}{2}}\left|y_{1}(t)\right|>0 .
$$

There is a constant $S, 0<S<\infty$, such that for all $t>b_{0}$ (say) we have $\left|y_{1}(t)\right|^{2} \geq S^{2}\{Q(t)\}^{-1}$. Multiply this inequality by the positive number $q(t)$ and integrate over $\left[b_{0}, b\right]$ to give, using (2.7),

$$
\begin{aligned}
& \int_{b_{0}}^{b} q(t)\left|y_{1}(t)\right|^{2} d_{\alpha} t \geq S^{2} \int_{b_{0}}^{b} \frac{q(t)}{Q(t)} d_{\alpha} t \\
& =\left.S^{2}\left[\ln Q\left(\frac{t^{\alpha}}{\alpha}\right)\right]\right|_{b_{0}} ^{b} \geq B S^{2} \ln \left(k \frac{b^{\alpha}}{\alpha}\right),
\end{aligned}
$$

where $B$ is a positive constant depending on $b_{o}$ and $k>0$. This implies that $q^{\frac{1}{2}} y_{1} \notin L_{\alpha}^{2}[0, \infty)$. This contradicts (2.10).Then there exists a sequence $\left\{b_{i} ; i \geq 1\right\}$, such that $b_{i} \rightarrow \infty$ as $i \rightarrow \infty$, and for which

$$
\lim _{i \rightarrow \infty} Q\left(b_{i}\right)^{\frac{1}{2}} y_{1}\left(b_{i}\right)=0
$$

From (2.15) and (2.14), we conclude that there is a sequence $\left\{b_{i} ; i \geq 1\right\}$ such that

$$
\lim _{i \rightarrow \infty} p\left(b_{i}\right) y_{1}\left(b_{i}\right) T_{\alpha} y_{2}\left(b_{i}\right)=0,
$$

for every pair $y_{1}, y_{2} \in D$. It now follows from (2.11) that (2.5) is satisfied for all $y_{1}, y_{2} \in D$. The proof is complete.

\section{CONFLicts OF INTEREST}

The authors declare that there are no conflicts of interest regarding the publication of this article.

\section{REFERENCES}

[1] Abdeljawad T., On conformable fractional calculus, J. Comput. Appl. Math. 279(2015), 57-66.

[2] Allahverdiev, B. P., Tuna, H., Yalçınkaya, Y., Conformable fractional Sturm-Liouville equation, Math. Meth. Appl. Sci., 42(10)(2019), 35083526.

[3] Baleanu, D., Jarad, F., Ugurlu, E., Singular conformable fractional sequential differential equations with distributional potentials, Quaest. Math. 42(3)(2018), 277-287.

[4] Everitt W. N., On the limit-point classification of second-order differential expressions, J. London Math. Soc. 41(1966), 531-534.

[5] Everitt W. N., On the limit-circle classification of second order differential expressions, Quart. J. Math. (Oxford) 2(23)(1972), 193-6.

[6] Hardy G. H., Littlewood J. E., Polya G., Inequalities, Cambridge University Press, New York, 1934.

[7] Khalil R. M., Al Horani ,Yousef A. and Sababheh M., A new definition of fractional derivative, J. Comput. Appl. Math. 264(2014), 65-70.

[8] Krein M. G., On the indeterminate case of the Sturm-Liouville boundary problem in the interval $(0, \infty)$, Izv. Akad. Nauk SSSR Ser. Mat., 16(4)(1952), 293-324 (in Russian).

[9] Levinson N., Criteria for the limit-point case for second order linear differential operators. Pest. Mat. Fys. 74(1949), 17-20.

[10] Miller, K. S., Ross, B., An Introduction to the Fractional Calculus and Fractional Differential Equation, Wiley Interscience, New York, 1993.

[11] Oldham K. B., Spainer J., The Fractional Calculus, Academic Press, New York, 1974.

[12] Podlubny I., 1999, Fractional Differential Equations, Mathematics in Science and Enginering, vol. 198, Academic Press, New York, London, Tokyo and Toronto.

[13] Ross B., Fractional Calculus and Its Applications, Springer, New York, 1975.

[14] Titchmarsh E. C., Eigenfunction expansions associated with second-order differential equations, Part I (2nd edition, Oxford University Press, 1962).

[15] Zhaowen, Z., Huixin, L., Jinming, C., Yanwei, Z., Criteria of limit-point case for conformable fractional Sturm-Liouville operators, Math. Meth. Appl. Sci. 43(2020), 2548-2557. 